Proc. XIX International School of Semiconducting Compounds, Jaszowiec 1990

\title{
ON DLTS EXPERIMENTS WITH EXTENDED DEFECTS*
}

\author{
WiKTOR SzKIElKo \\ Institute of Physics, Polish Academy of Sciences, Al. Lotników 32/46, 02-668 \\ Warszawa, Poland \\ (Received August 8, 1990)
}

It is shown that the line shape of DLTS spectra measured in silicon with a high concentration of extended defects can be consistently explained if the both processes, thermal emission and tunneling of majority carriers from charged defects, are taken in to account.

PACS numbers: 71.55.-i

\section{Introduction}

Characterization of dislocations in semiconductors by their electronic levels remains an open question. In order to find out which of traps are really connected with dislocation cores, DLTS measurements were performed in silicon crystals dislocated under various conditions of plastic deformation [1], and crystals which after a deformation were subjected to various annealing treatments [2] or hydrogenated [3]. The another approach to the problem is concentrated on investigations of physical phenomena under DLTS experiments in dislocated samples which seem to be characteristic of dislocations. A logarithmic dependence of a DLTS signal has been claimed to be characteristic of electron states within extended defects [4]. Theoretical modifications of emission and capture rates at dislocation states and their influence on the shape of a DLTS line have been studied in [5]. The further attempt to explain the shape of DLTS spectra is given in the present work.

*This work was supported by CPBP 01.04 . 


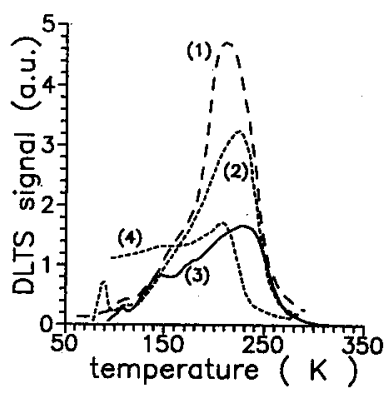

Fig. 1. DLTS spectra of extended defects in silicon; (1) $60^{\circ}$-dislocation introduced at $680^{\circ} \mathrm{C}$; (2) the same after annealing at $800^{\circ} \mathrm{C}$ [7]; (3) dislocated $\mathrm{Si}$, high stress deformation [1]; (4) Si with $10^{7} \mathrm{~cm}^{-1}$ oxidation induced stacking faults.

\section{DLTS spectra of extended defects}

In all so far reported DLTS data obtained in dislocated silicon one can distinguish two portions of the spectrum. The one consists of peaks whose number as well as positions and amplitudes vary depending on the deformation conditions. The another portion, that is further called "extended line", forms a wide featureless spectrum. The signal amplitude in the extended line increases monotonously with temperature ending with a relatively abrupt shoulder on the high temperature side. In Fig. 1 there are some DLTS spectra with a strongly pronounced extended line. The curves (1)-(3) were measured in Si samples dislocated in various ways. The curve (4) presents the spectrum measured in a silicon sample with a high concentration (about $10^{7} \mathrm{~cm}^{-2}$ ) of $2-4 \mu \mathrm{m}$ long stacking faults and a very low concentration of dislocation [6].

The characteristic feature of any DLTS spectrum measured in silicon with extended defects is a logarithmic dependence of the line amplitude on the filling pulse duration. In samples with dominating extended DLTS lines also logarithmic kinetics of electron emission from traps has been observed [8].

\section{Carrier capture and emission at traps within extended defects}

Filling electron states within extended defects with majority carriers builds up a repelling potential barrier at the defects. The formula for the electron capture rate at dislocation states, $r_{\mathrm{e}}$, which accounts for the repelling barrier has been given in [9]:

$$
r_{\mathrm{e}}=c_{\mathrm{e}} n_{0}(1-f) \exp (-\phi f),
$$

where $c_{\mathrm{e}}$ is the capture coefficient, $n_{0}$ is the concentration of electrons and for those which can be captured it is reduced by the exponential factor with the term $\phi f$ - the height of the potential barrier expressed in units of $k_{\mathrm{B}} T\left(k_{\mathrm{B}}-\right.$ Boltzmann constant, $T$ - temperature, $f$-the occupancy of dislocation states). This capture 


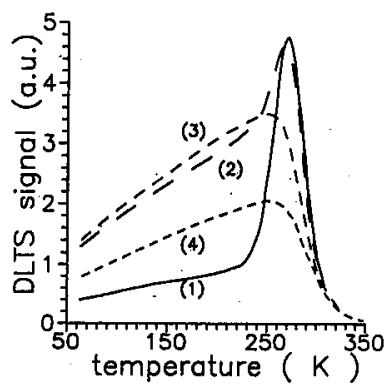

Fig. 2. Simulated DLTS spectrum of dislocations with $0.54 \mathrm{eV}$ deep traps; (1)-(4) increasing portion of tunneling emission.

rate gives a nearly logarithmic dependence of the amplitude of the DLTS line on the duration of the filling pulse. In order to account for observed nonlinearities in the emission kinetics the following expression is proposed for the electron emission rate:

$$
g_{\mathrm{e}}=c_{\mathrm{e}} n_{1} f \exp (\eta f)
$$

where $n_{1}=N_{\mathrm{C}} \exp \left(-\Delta \epsilon_{T}\right)$ is the standard parameter of the Shockley-Read recombination theory ( $N_{\mathrm{C}}$ is the density of states in the conduction band and $\Delta \epsilon_{T}$ is the depth of the trap state measured in units of $k_{\mathrm{B}} T$ ). The above expression differs from the standard one by the exponential factor $\exp (\eta f)$, where $\eta f$ is equal to the energy of electrostatic interaction between neighboring electrons trapped on dislocation centers. This energy can be regained during the thermal emission process by rearranging the distribution of electrons at the dislocation. The main effect of such a modification in the emission rate is apparent reducing the trap depth by $\eta f$. Using equations (1) and (2) for the trapping-emission kinetics requires numerical simulation of a DLTS experiment. Similar modifications of the trapping and emission rates have been proposed by Schröter et al. [5]. From their results as such one can infer that the postulated nonlinearities cannot give any extended DLTS lines.

The assumption made here is that electrons trapped at extended defects are mobile within the defect or at least within a macroscopic segment of the defect. As a consequence trapped electrons can adjust their distribution to the external field in the semiconductor junction increasing locally the occupation factor $f$ almost to unity.

In silicon this effect can give an apparent reduction of the trap depth by up to $\eta f k_{\mathrm{B}} T=0.33 \mathrm{eV}$. For traps found in experiments as deep as $0.55 \mathrm{eV}$ even that is not enough to cancel the exponential dependence of the emission rate on temperature.

On the other hand such an increase in $f$ makes the tunneling of trapped electrons a highly probable process. More, the tunneling process is not thermally activated. Thus a DLTS signal appears at any temperature provided there is a 
component of tunneling rate falling into the rate window of the equipment and the trap filling rate is high enough. Tunneling from dislocation states has been discussed in [11] in order to explain the mechanism of electronic transport along dislocation cores in silicon diodes.

Figure 2 shows the results of computer simulation of the DLTS experiment in silicon with dislocation electron traps with single energy level at $0.54 \mathrm{eV}$ below the conduction band. The traps are emptied in course of the two competitive processes: thermal excitation and tunneling. The tunneling rate is taken as a parameter. If in course of the DLTS period the traps discharge only little by tunneling then a well resolved DLTS line can be seen (curve (1)). With increasing tunneling rate the amplitude of the extended line increases while the amplitude of the sharp line decreases and it broadens apparently: (2). With further increase of the tunnel rate the sharp line disappears completely and only the extended line can be seen (curves 3 and 4). It is worth noting that in the present model the shape of the low-temperature side of this line reflects the temperature dependence of the trapping rate.

\section{Conclusions}

The DLTS spectra observed experimentally in silicon crystals with extended defects can be easily simulated assuming the two competitive processes for emission of trapped carriers from the extended defects, namely, thermal excitation and tunneling. The proposed model can be experimentally verified by investigations of the nonstationary transport via dislocation states in semiconductor junctions.

\section{References}

[1] E.R. Weber, H. Alexander, J. Phys. 44, C4-319 (1983).

[2] J.R. Patel, L.C. Kimmerling, J. Phys. 40, C6-67 (1979).

[3] B. Pohoryles, J. Phys. 44, C4-359 (1983).

[4] W. Szkiełko, O. Breitenstein, R. Pickenhein, Cryst. Res. Technol. 16, 197 (1981);

N.A. Yarykin, O.V. Feliksova, in Defects in Crystals, Proc. 7th Internat. School, Szczyrk (Poland) 1988, ed. E. Mizera, World Scientific, Singapore 1988, p. 366.

[5] W. Schröter, I. Queisser, J. Kronewitz, in Structure and Properties of Dislocations in Semiconductors 1989, Inst. Phys. Conf. Ser. No. 104, eds. S.G. Roberts et al., Institute of Physics, Bristol 1989, p. 75.

[6] G.R. Lahiji, A.R. Peaker, B. Hamilton, in Structure and Properties of Dislocation in Semiconductors 1989, Inst. Phys. Conf. Ser. No. 104, eds. S.G. Roberts et al., Institute of Physics, Bristol 1989, p. 239.

[7] J. Kronewitz, W. Schröter, Izv. Akad. Nauk, USSR Ser. Fiz. 51, 682 (1987).

[8] M. Seibt, Diploma Work, University of Gőttingen (1982).

[9] T. Figielski, Phys. Status Solidi 9, 555 (1965). 
[10] W.T. Read, Philos. Mag. 7, 775 (1954).

[11] R. Nitecki, B. Pohoryles, Appl. Phys. A 36, 55 (1985);

B. Pohoryles, Acta Phys. Pol. A69, 397 (1986). 